\begin{tabular}{|c|l|}
\hline Title & Development of a high-quantum-efficiency single photon counting system \\
\hline Author(s) & Takeuchi, Shigeki; Kim, Jungsang; Y amamoto, Y oshihisa; Hogue, Henry H. \\
\hline Citation & $\begin{array}{l}\text { A pplied Physics Letters, 74(8), 1063-1065 } \\
\text { https://doi.org/10.1063/123482 }\end{array}$ \\
\hline Issue Date & 1999-02-22 \\
\hline Doc URL & http://hdl.handle.net/2115/5564 \\
\hline Rights & Copyright $\odot$ 1999 A merican Institute of Physics \\
\hline Type & article \\
\hline File Information & APL 74-8.pdf \\
\hline
\end{tabular}

Instructions for use 


\title{
Development of a high-quantum-efficiency single-photon counting system
}

\author{
Shigeki Takeuchi ${ }^{\mathrm{a})}$ \\ JST-PRESTO "Field and Reactions,"' A.T.R.C. Mitsubishi Electric Corporation, Amagasaki, \\ Hyogo 661-8661, Japan \\ Jungsang Kim and Yoshihisa Yamamoto \\ ERATO Quantum Fluctuation Project, E. L. Ginzton Laboratory, Stanford University, Stanford, \\ California 94305-4085
}

Henry H. Hogue

Research and Technology Center, Boeing North American, Anaheim, California 92803

(Received 31 August 1998; accepted for publication 18 December 1998)

\begin{abstract}
A high-quantum-efficiency single-photon counting system has been developed. In this system, single photons were detected by a visible light photon counter operated at $6.9 \mathrm{~K}$. The visible light photon counter is a solid state device that makes use of avalanches across a shallow impurity conduction band in silicon. Threefold tight shielding and viewports that worked as infrared blocking filters were used to eliminate the dark count caused by room-temperature radiation. Corrected quantum efficiencies as high as $88.2 \% \pm 5 \%$ (at $694 \mathrm{~nm}$ ) were observed, which we believe is the highest reported value for a single-photon detector. The dark count increased as the exponential of the quantum efficiency with changing temperature or bias voltage, and was $2.0 \times 10^{4} \mathrm{cps}$ at the highest quantum efficiency. (C) 1999 American Institute of Physics. [S0003-6951(99)01308-X]
\end{abstract}

Photon counting methods have been widely applied to the accurate measurement of very weak lights, and have recently been used in quantum key distribution systems which encode a data bit to a single photon. The sensitivity of the measurement and the data transmission efficiency depends on the quantum efficiency of photon counters. Therefore, improvements in quantum efficiency have been the main issue in the development of photon counters. Photomultiplier tubes (PMTs) have been commonly used as the photon counter, however, their quantum efficiency is $25 \%$ at its most sensitive wavelength and $15 \%$ in the near infrared region. Silicon avalanche photodiodes (APDs) operating in Geiger mode have become popular, and a quantum efficiency of $76 \%$ was recorded with the APD, ${ }^{1}$ which is the highest value so far. One of the important applications for such a highquantum-efficiency photon counter is the loophole free test of Bell's inequality. ${ }^{2-5}$ In order to close the loophole, the quantum efficiency of the system has to be higher than $83 \%$ when an ordinary Einstein-Podorsky-Rosen (EPR) pair source is used. ${ }^{6,7}$ The loophole free test has not been performed yet, and the lack of a highly efficient single-photon counting system over the threshold is one of the reasons.

The visible light photon counter (VLPC) is an alternative detector, which is a solid state device using the avalanche multiplication effect of electrons in an impurity band in silicon. ${ }^{8-10}$ The quantum efficiency of a VLPC was estimated to be $93 \%$, but the measured value as a system was less than $70 \%$. $^{1}$ The difficulty was that, in order to cope with both "high quantum efficiency" and "low dark counts," a special shielding system which has high transmittance for the desired wavelength but filters out room-temperature radiation sufficiently is required. Infrared photons of the radiation up to $28 \mu \mathrm{m}$ in wavelength can excite electrons in the shal-

${ }^{a)}$ Electronic mail: takeuchi@bio.crl.melco.co.jp low impurity band and might cause large dark count (up to $\left.10^{15} \mathrm{cps}\right)$.

We developed the cryostat system shown in Fig. 1. Threefold shields at 77,4 , and $6.5 \mathrm{~K}$ were used to reduce the thermal photons reaching the detector. We put felt between the shields to block the background photons passing through the shields. For the viewports on the shields, we used antireflection coated acrylic plates which attenuate the thermal radiation (by more than $10^{-14}$ in total) but transmit visible photons with high efficiency (97\% at $694 \mathrm{~nm}$ in total). In order to measure the quantum efficiency of the system, a cw light at $694 \mathrm{~nm}$ from the laser diode was attenuated by neutral density (ND) filters and directly focused onto the VLPC from outside through the viewports. The reflected photons at the VLPC surface $(15.6 \%$ at $694 \mathrm{~nm})$ were recollected by a spherical refocusing mirror (99\% reflectance) inside the cryostat. The signal was amplified by a homemade amplifier with a bandwidth of $40 \mathrm{MHz}$ which was installed in the cryostat. The observed FWHM of the signal pulse was $40 \mathrm{~ns}$.

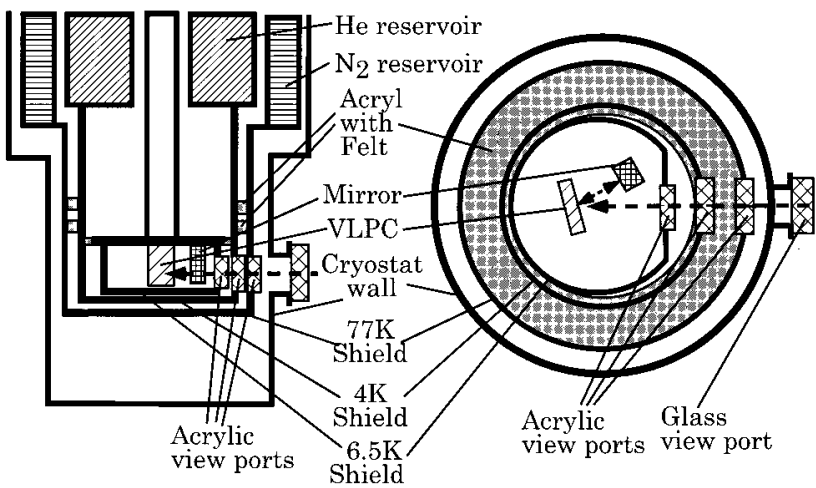

FIG. 1. Schematic diagram of the cryostat system with threefold thermal radiation shields. 




FIG. 2. Measured quantum efficiency vs bias voltage for various temperatures. The error bars which should appear on the dots are omitted for simplicity except for $6.91 \mathrm{~K}$.

To account for the missed pulses due to the dead time $\tau$, the corrected counts ( $N^{\prime}$ counts per second or cps) is estimated as follows:

$$
N^{\prime}=\frac{N}{1-N \tau},
$$

where $N$ is the observed counts. Here $\tau$ was estimated to be 100 ns by saturation measurement. Therefore, the corrected quantum efficiency $Q$ is given by

$$
Q=\left(\frac{N_{S}}{1-N_{S} \tau}-\frac{N_{b}}{1-N_{B} \tau}\right) / N_{\text {in }} .
$$

In the equation, $N_{S}$ is the observed count with input signal photons and $N_{B}$ is the observed dark-count without input photons. In such a definition of quantum efficiency, the effect of detector saturation is also accounted for. The number of incident photons $N_{\text {in }}$ was calculated by $N_{\text {in }}=P \lambda /(c h)$, where $\lambda$ is the wavelength $(694 \mathrm{~nm}), c$ is the speed of light, $h$ is Planck's constant, and $P$ is the power of light incident to the VLPC. $P$ is estimated by $P=\alpha P_{0}$, where $P_{0}$ is the power of the diode laser output measured by a power meter (Coherent Fieldmaster with LM-2 Head) which had a 3\% uncertainty, and $\alpha$ is the total attenuation given by the ND filters which has a $1 \%$ uncertainty. The total attenuation was calculated by simply multiplying the measured attenuation of each ND filter. We carefully avoided the multireflection of the incident laser beam between the attenuators. The observed counts also had a 1\% standard deviation.

Figure 2 shows the quantum efficiency measured as a function of the operation temperature and bias voltage. The highest quantum efficiency of $88.2 \% \pm 5 \%$ at $694 \mathrm{~nm}$ was observed with a bias voltage of $7.3 \mathrm{~V}$ at $6.91 \mathrm{~K}$, which we believe is the highest single-photon detection efficiency reported so far. The residual reflection on the surfaces of the windows is estimated to be $3.1 \%$. Even with a refocusing mirror of $99 \%$ reflectance, $2.4 \%$ of the incident photons were missed because of the second reflection at the VLPC surface. We measured the power of laser beam through a pinhole which had the size of the VLPC surface area $(1.0 \mathrm{~mm}$ in diameter) at the focal point and estimated that $1.5 \%$ of the photons were focused outside the sensitive area of the VLPC. ${ }^{11}$ Considering these losses, we estimate the internal quantum efficiency of VLPC itself to be $94.7 \% \pm 5 \%$. This number is consistent with the rough estimate of the total absorption probability of a photon in the VLPC structure. At this operating point, the dark count was $2.0 \times 10^{4} \mathrm{cps}$. Less than $500 \mathrm{cps}$ of the dark count was caused by roomtemperature thermal photons leaking through the viewports, as was confirmed by covering each of the viewports on the radiation shields with reflective metal tape. Most of the observed dark count was estimated to be intrinsic to the detector. In the case of other devices, afterpulsing sometime causes wrong estimates of the quantum efficiency. Several tests have been made for VLPCs, and no afterpulsing has ever been observed. ${ }^{12}$

The operation principles of a VLPC are as follows. ${ }^{13}$ The VLPC structure contains two active layers, an undoped silicon layer overlying a moderately arsenic doped "gain" layer. This structure is provided with top and bottom electrical contacts. A photon incident through the top contact is absorbed in the undoped layer, creating an electron-hole pair. Under the applied bias, the electron drifts to the top contact and is collected, while the hole is injected into the gain layer. In the gain layer, the hole encounters and ionizes a neutral arsenic atom, producing an electron and a mobile ionizeddonor charge $\left(D^{+}\right)$. The electron from the gain-layer ionization is accelerated by the electric field, initiating a cascade of secondary ionization by the impact on additional neutral arsenic atoms. A pulse of several thousand electrons results from each primary ionization event. A like number of $D^{+}$ charges are created in the gain layer. These must drift out of the gain layer to the back contact before an avalanche can be repeated in the same small portion of the VLPC gain layer.

As shown in Fig. 2, the quantum efficiency (probability of producing an ionization pulse) increases with the bias voltage. At low bias, only a small fraction of the electrons produced in the gain layer are able to obtain sufficient energy from the electric field to initiate an avalanche before they move into the undoped layer. At higher voltages almost all the gain-layer-generated electrons can attain sufficient energy to initiate avalanches. The QE vs bias curve shifts to a higher voltage as the temperature decreases. This is because the deepest part of the gain layer acts like a large series resistor, which becomes more resistive as the temperature decreases. This series resistance reduces the effective bias applied to the active part of the gain layer where the avalanches are produced. When we increased the bias voltage at temperatures above $7 \mathrm{~K}$, the quantum efficiency reached $88 \%$ with the bias voltage below $7.3 \mathrm{~V}$ and saturated. The device went into a breakdown state when the bias voltage was increased above $7.3 \mathrm{~V}$. The breakdown voltage did not depend strongly on the temperature.

Similar to the quantum efficiency, the dark count also showed a strong dependence on the bias voltage and temperature. As shown in Fig. 3, we found that the dark count of the VLPC increased roughly as the exponential of the quantum efficiency with the changing temperature or bias voltage. When the corrected quantum efficiency $Q$ is less than $85 \%$ and the measured dark counts $N_{B}$ is less than $10^{4} \mathrm{cps}$, this relation can be described well by the following equation: 


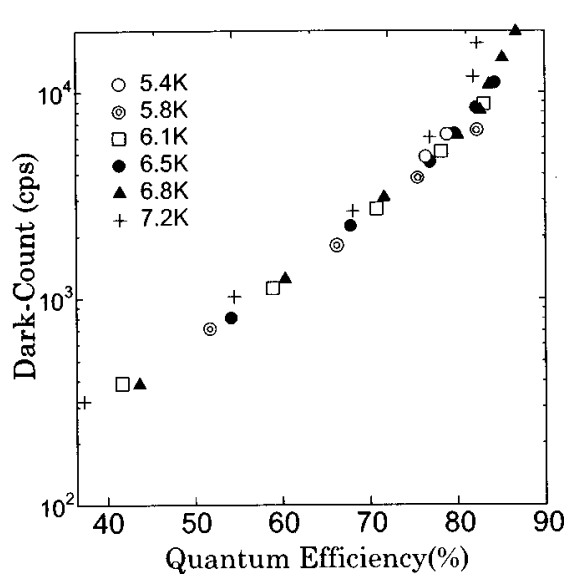

FIG. 3. Measured dark count vs the quantum efficiency for various temperatures.

We also measured the saturation effect of the detector response by changing the number of incident photons $N_{\text {in }}$. We fixed the spot size of the focused beam so that we could obtain a maximum count rate when $N_{\text {in }}$ was $2 \times 10^{5} \mathrm{cps}$. Equation (1) with the dead-time $\tau=100 \mathrm{~ns}$ fitted well to the measured counts with $N_{\text {in }}$ from $10^{4}$ to $2 \times 10^{7} \mathrm{cps}$. When $N_{\text {in }}$ was smaller than $2 \times 10^{5} \mathrm{cps}$, the degradation of quantum efficiency due to saturation effect was smaller than $1 \%$. Since a similar saturation was observed when we used a wide-band amplifier $(500 \mathrm{MHz})$ to decrease the signal pulse width to $2 \mathrm{~ns}$, the saturation is attributed to the slow movement of the $D^{+}$charge to the contact region rather than the overlap of the signal pulses. The quantum efficiency was also degraded when the beam was focused into too small an area. When $N_{\text {in }}$ was $10^{6} \mathrm{cps}$, we could minimize the degradation at $7 \%$ by the optimum choice of the focused beam size.

Our system will find a lot of applications, including fundamental tests of quantum mechanics like Bell's inequality test, and quantum information experiments like quantum cryptography and quantum teleportation. Let us discuss briefly the application of the system to a detection loophole free test of Bell's inequality. The required quantum efficiency for the test depends on $R_{b}$, the ratio of the dark count to the signal count. ${ }^{6}$ The number of incident photons should be increased in order to decrease $R_{b}$, but the effective quantum efficiency rapidly decreases if the incident photon number exceeds $1 / \tau$. We found that if the quantum efficiency of the system is larger than $92 \%$, which should be realized by improving the optical loss in the present system, we will be able to perform a detection loophole free test with $10^{6} \mathrm{cps}$ photon pairs in an EPR state with optimum nonequal probability amplitudes. ${ }^{14}$

In some applications, a wide surface area $(1 \mathrm{~mm}$ in diameter) of the detector and high counting rate (up to $10^{7} \mathrm{cps}$ or more) may be advantageous. The VLPC features high quantum efficiency between $400 \mathrm{~nm}$ and $1 \mu \mathrm{m}$ with a maximum at $540 \mathrm{~nm}$ where the antireflect coating is optimized. On the further improvement of VLPCs, for example, devices with dark count rates as low as a few counts per second should be achievable with the acceptance of smaller saturation level (up to $10^{4} \mathrm{cps}$ ). The saturation rate and the dark count rate seems to be in a trade-off relation such that the increase of carrier mobility for the high saturation rate causes an increase in the dark-count.

In summary, a photon counting detection system with a high quantum efficiency of $88.2 \% \pm 5 \%$ was achieved using the VLPC. The dark count of the detector increased roughly as the exponential of the quantum efficiency as the temperature or bias voltage changed, and was $2.0 \times 10^{4} \mathrm{cps}$ at the highest quantum efficiency.

The authors wish to thank the Yamamoto group members at Stanford, Dr. M. G. Stapelbroek at Boeing, and Dr. T. Isu at Mitsubishi Electric for their helpful discussions.

${ }^{1}$ P. G. Kwiat, A. M. Steinberg, R. Y. Chiao, P. H. Everhard, and M. D. Petroff, Phys. Rev. A 48, R867 (1993).

${ }^{2}$ A. Aspect, P. Grangier, and G. Roger, Phys. Rev. Lett. 49, 460 (1981).

${ }^{3}$ Z. Y. Ou and L. Mandel, Phys. Rev. Lett. 61, 50 (1988).

${ }^{4}$ J. F. Clauser and A. Shimony, Rep. Prog. Phys. 41, 1881 (1978).

${ }^{5}$ E. Santos, Phys. Lett. A 212, 10 (1996).

${ }^{6}$ When we use an EPR state where entangled states are superposed in equal amounts, the quantum efficiency should be larger than $82.8 \%$. When we use an EPR state with nonequal amounts, and the ratio of background counts to signal counts is 0 , the quantum efficiency required can be lowered to 66.7\%. P. H. Eberhard, Phys. Rev. A 47, R747 (1993).

${ }^{7}$ P. G. Kwiat, K. Mattel, H. Weinfurter, and A. Zeilinger, Phys. Rev. Lett. 75, 4337 (1995).

${ }^{8}$ M. D. Petroff, M. G. Stapelbroek, and W. A. Kleinhans, Appl. Phys. Lett. 51, 406 (1987).

${ }^{9}$ G. B. Turner, M. G. Stapelbroek, M. D. Petroff, E. W. Atkins, and H. H. Hogue, in Proceedings of the Workshop on Scintillating Fiber Detectors, Notre Dame University, 24-28 October, 1993, edited by R. Ruchti (World Scientific, Singapore, 1994), p. 613.

${ }^{10}$ J. Kim, Y. Yamamoto, and H. H. Hogue, Appl. Phys. Lett. 70, 2852 (1997).

${ }^{11}$ As described later, the spot size of the focused laser beam was intentionally enlarged in order to avoid the saturation.

${ }^{12} \mathrm{H}$. H. Hogue (unpublished).

${ }^{13}$ M. Atac, J. Park, D. Cline, D. Chrisman, M. Petroff, and E. Anderson, Nucl. Instrum. Methods Phys. Res. A 314, 56 (1992).

${ }^{14}$ The brightest EPR source used in the previous experimental test of Bell's inequality was only $1500 \mathrm{cps}$. Therefore, the attempts to invent a brighter source are important. P. G. Kwiat, J. Mod. Opt. 44, 2173 (1997). 